SECCIÓN VARIA 



\title{
A DATAÇÃO PELO RADIOCARBONO DE ELEMENTOS DE RODAS ROMANAS DE MADEIRA PARA ELEVAÇÃO DE ÁGUA NAS Minas DE Rio Tinto
}

\author{
Aquilino Delgado Domínguez \\ Fundación Riotinto \\ António M. Monge SoARes \\ ITN/IST Instituto Superior Técnico. Universidade Técnica de Lisboa \\ Paula F. QueIroz
}

Terra Scenica. Centro para a criatividade partilhada das Ciências, Artes e Tecnologias

Recibido: 24/01/2013

Revisado: 06/02/2013
Aceptado: 07/02/2013

Publicado: 17/06/2013

\section{RESUMO}

Fragmentos de madeira dos raios das rodas utilizadas no sistema de drenagem das minas romanas de Rio Tinto e que se encontram depositados no Museu Mineiro de Rio Tinto foram datados pelo radiocarbono. Todos os fragmentos datados foram identificados como de madeira de sobreiro (Quercus suber) e as datas obtidas foram consideradas como terminii post quem para o fabrico e utilização das referidas rodas. Assim, estas serão atribuíveis aos Sécs. I a III d. C.

\section{Palavras Chave}

Rodas romanas de elevação de água, Minas de Riotinto, Sobreiro, Datação pelo radiocarbono.
ABSTRAC

Wood remains from spokes of Roman water wheels discovered in the Rio Tinto mines (Southern Spain) and currently deposited at the Mines Museum of Rio Tinto were radiocarbon dated. Five samples identified as wood from corkoak (Quercus suber) were processed providing post quem dates for the manufacture and use of the wheels. In this way, these Roman wheels must be ascribed to a period between the first and the third century A.D.

\section{KEYWORDS}

Roman water wheels, Rio Tinto mines, Corkoak, Radiocarbon dating. 
La noria (rota aquaria) fue una de las máquinas más empleadas en época romana para desaguar el interior de las minas (Luzón Nogué, 1968). Restos de estos artilugios se han documentado en Britania (Davies, 1935; Jones et al., 1960; Lewis, 1977), en Dacia (Wollmann, 1996; Slotta et al., 2002), y en Hispania, donde los hallazgos han sido numerosos en los distritos mineros del suroeste, en São Domingos (Domergue et al.,1999), Tharsis (Stevenson, 1875; Domergue y Bordes, 2004), y Riotinto (Delgado y Regalado, 2010). Sólo en Riotinto se han registrado una cincuentena de ruedas hidráulicas (Ver plano 1), de las que actualmente se conserva una completa y tres mitades de discos y algunos ejes de bronce en el Museo Provincial de Huelva, y un tercio de otra en el Museo Británico de Londres (Weisberger, 1979). En el Museo Minero de Riotinto se conservan también un eje de madera y 387 fragmentos de madera de noria de los ejemplares hallados en Filón Sur en 1922. Las analíticas que se presentan en este trabajo se han realizado sobre una muestra de estas maderas.

Estas maderas fueron depositadas tras su hallazgo en el Museo de Bella Vista, propiedad de Río Tinto Co. Ltd., en la artesa $n^{0} 3$ bajo la vitrina central, donde gracias al trabajo de William conocemos que ya estaban ubicadas en 1932. Con la creación del Museo Provincial de Huelva en 1973 muchos de los materiales de esta colección fueron trasladadas a Huelva, entre ellos la noria ecnontrada en masa Planes en 1928, los ejes de bronce plomado hallados en Filón Sur, etc., pero estos fragmentos de madera

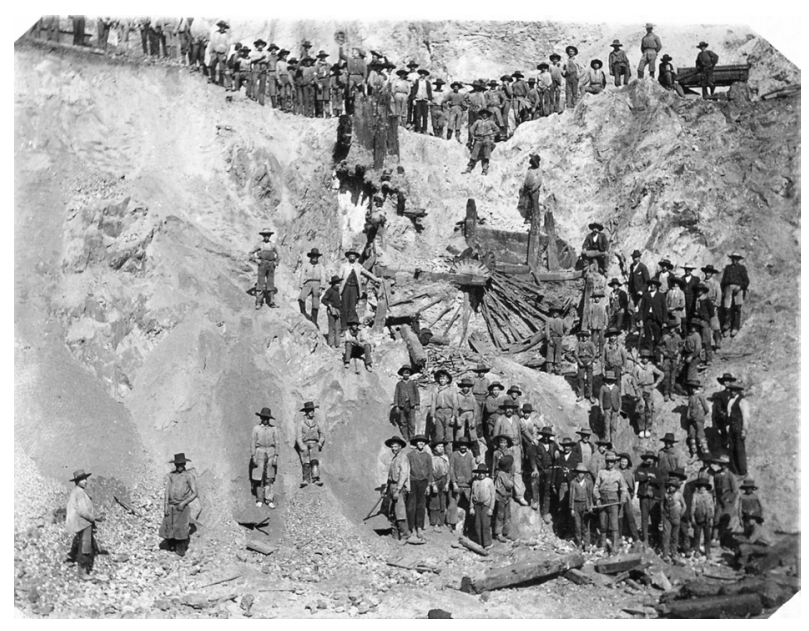

Figura 1 Norias romanas halladas en Filón Sur (Río Tinto) en 1910. Foto A-3/277. Archivo de Fundación Río Tinto (AFRT). permanecieron en Riotinto, hasta que en 1987 fueron trasladados al Museo Minero de Riotinto, junto con el resto de materiales que permanecían en el Museo de Bellavista (Delgado y Regalado 2010, 670).

El hallazgo DE NORIAS ROMANAS EN EL FILÓN SUR DE Riotinto.

A fines de 1910, durante los trabajos mineros que se llevaban a cabo en la zona centro del Filón Sur o Nerva, se documentó un sistema de 14 parejas de norias, es decir 28 (Palmer 1926-1927) (Fig. 1). Presentaban tan mal estado de conservación que no se pudo "recuperar" ninguna. Sus tamaños variaban entre los 4,648 metros y los 4,52 metros.

En una zona cercana, a una cota superior, se produjo otro hallazgo entre 1919 y 1921, ocho parejas de norias según Palmer (Palmer, 1926-1927, 299336), y once según Williams (Williams, 1932,18), quien corrobora la situación, “...During the year 1919 to 1921, a whole nest of similar wheels was uncovered in the South Lode..." (Williams 1932, 3), y nos aporta el dato de que fueron halladas en la zona próxima a la galería de desagüe de San Luís (Williams, 1932,18). Tenían unas dimensiones medias de 4,65 metros y disponían de treinta cangilones. Estaban ubicadas de forma escalonada, cada pareja elevaba el agua desde el fondo de la cámara donde se encontraba hacia los canales en la parte superior que comunicaban con la cámara superior, donde se encontraba la siguientes norias del sistema, y así de muro a techo.

R. E. Palmer calculó que una rota como éstas halladas en Filón Sur podría elevar estaría en torno a los 18,9 galones de agua por minuto, equivalentes a $0,083 \mathrm{~m}^{3}$ u 83,252 litros por minuto, y que la fuerza necesaria para moverla sería de 2270 pies libras por minuto, equivalentes a 0,07 caballos de vapor métricos (Palmer 1926-1927, 299-336).

Estas cadenas de norias halladas entre 1910 y 1921 formarían parte de un mismo sistema, como se recoge en el plano de Williams (Williams 1932, 18 plate 1), en el que se señala que "Chain of 14 wheels feeding to lowest Wheel of Chain " $A$ "'. Es decir, el sistema 28 ruedas hidráulicas halladas en el último trimestre de 1910 subiría el agua desde los 256.2 metros sobre el nivel del mar hasta la cota 308 metros, donde la parte inferior del la cadena de 14 norias documentadas entre 1919 y 1921 subía el agua 29,6 metros, hasta la galería romana de desagüe de San Luís, al pie del Cerro de las Vacas. De esta forma este sistema desaguaba la zona central de 
Filón Sur elevando el agua 81,4 metros (Fig. 2), desde los niveles inferiores de la zona de laboreo minero romano hasta la galería inclinada del Cerro de las Vacas (Delgado y Regalado, 2010a), desde donde el agua caía por su propio peso hacia el valle del río Agrio, que desagua tras un corto trecho en el río Tinto. Esta galería de desagüe romana fue empleada desde 1725 hasta el último tercio del siglo XIX como punto de aprovisionamiento de agua para las labores hidrometalúrgicas de cementación natural (Ezquerra del Bayo, 1852, 31).

La construcción de un sistema de desagüe como éste, con más de cuarenta norias para subir el agua a una altura de ochenta metros, hasta alcanzar la galería inclinada de San Luis, precisaría una fuerte inversión en trabajo y capital, para la maquinaria de madera, para el trazado de las labores de las cámaras y de los canales y su fortificación, y para el mantenimiento, que sería más costoso que la construcción, ya que las labores de desagüe no podían detenerse, pues se inundarían las zonas de arranque y ello podía ocasionar el fin de la extracción.

Este sistema de norias fue el primero documentado en las zonas de explotación de cobre de época romana. En 1928 se hallaron dos ruedas hidráulicas más en otro de los sectores de minería romana de cobre, en masa Planes (Pérez et al., 2012, 53 y 57). Antes de estas fechas los restos de norias sólo habían aparecido en Filón Norte, el sector de minería romana argentífera, a una profundidad de 12 metros. Los minerales que contenían cantidades de plata beneficiables se ubicaban en la zona de oxidación (gossan), por lo que no era necesario un trabajo de desagüe a tanta profundidad.

En época Julio-Claudia la minería había estado dirigida preferentemente a la producción argentífera, y esto nos lleva a pensar que las norias de Filón Sur, que suponen un interés especial en la minería del cobre, pueden reflejar una importante potenciación de la minería cuprífera a partir de época trajano-hadriananea. A esta época pertenece también una regulación que expresa ese impulso a la minería del cobre, las tablas de bronce de Vipasca (Aljustrel, Portugal), un coto minero que se encuentra entre las minas de cobre más importantes del Imperio.

\section{IDENTIFICAÇÃO BOTÂNICA DE AMOSTRAS DE MADEIRA.}

Foi analisado um conjunto de cinco amostras de madeira que foram, posteriormente, objecto de datação pelo radiocarbono. Todas estas amostras eram fragmentos de raios de rodas de elevação de água

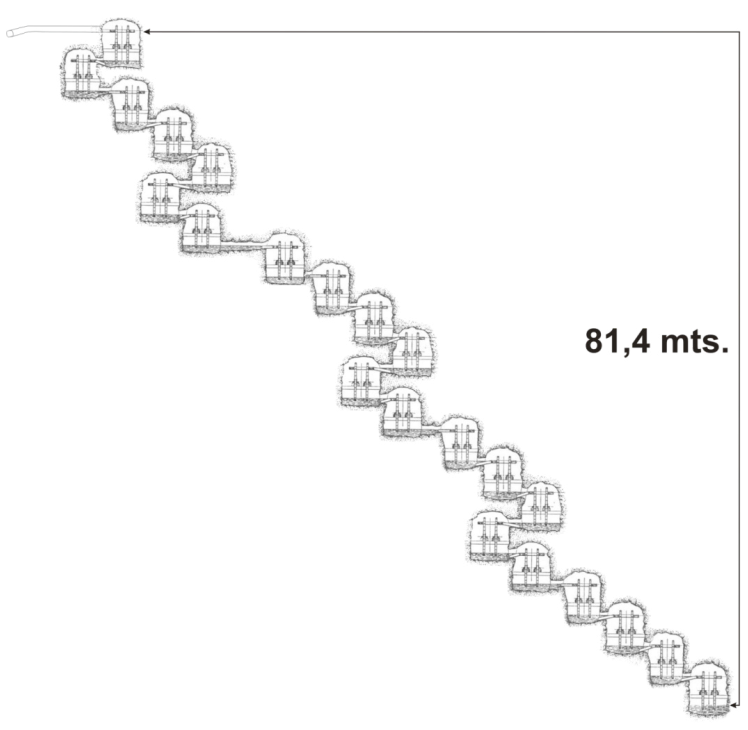

Figura 2 Recreación del sistema de 44 norias de Filón Sur con los datos aportados por Palmer y el plano de WILLIAMS, D. 1932, Plate 1, Pag. 18.

das minas romanas de Rio Tinto e encontravam-se depositadas no Museu Mineiro de Rio Tinto que foram tomados nos anos 90 do século XX.

Os fragmentos de madeira foram hidratados com água fervente durante várias horas, sendo, em seguida, realizados cortes finos com bisturi, segundo as três secções de diagnóstico - transversal, radial e tangencial - e observados e diagnosticados com microscopia óptica de transmissão. Não foi, no entanto, possível a realização de cortes finos da secção transversal, dado que implicaria a utilização de técnicas de impregnação e consolidação da madeira (o que não era admissível tendo em conta a sua utilização posterior como amostras a datar pelo radiocarbono). Assim, a observação e o diagnóstico dos padrões de porosidade e frequência/dimensão de raios multisseriados foram realizados com o auxílio da lupa binocular. A identificação da madeira constituinte das amostras foi auxiliada por material de referência e bibliografia especializada (Schweingruber, 1990a; 1990b).

$\mathrm{O}$ resultado das análises encontra-se descrito a seguir (Fig. 3):

Secção transversal - camadas de crescimento distintas; porosidade semidifusa; poros pouco frequentes (ocasionalmente mais frequentes em Muestra 2 e Muestra 5), dispostos em filas radiais, com um ou dois poros maiores concentrados no início do anel de crescimento. Os poros vão diminuindo gradualmente de diâmetro do início 
para o fim da camada de crescimento. Poros com tilos. Raios multisseriados muito largos e muito frequentes.

Secção tangencial - raios unisseriados abundantes, homogéneos, formados por células circulares a elípticas.

Secção radial - raios homogéneos, formados exclusivamente por células prostadas. Vasos grandes, densamente pontuados, com tilos. Pontuações intervasculares grandes, circulares. Pontuações ra- diovasculares grandes, opostas, alongadas radialmente. Placas de perfuração simples.

Todos os fragmentos observados foram identificados como madeira de sobreiro (Quercus suber). Embora dois fragmentos apresentassem uma frequência de poros mais elevada que o padrão típico de $Q$. suber (aproximando-se, assim, da morfologia apresentada pela madeira de azinheira $-Q$. rotundifolia), a clara variação de diâmetro dos poros ao longo da camada de crescimento e a presença de
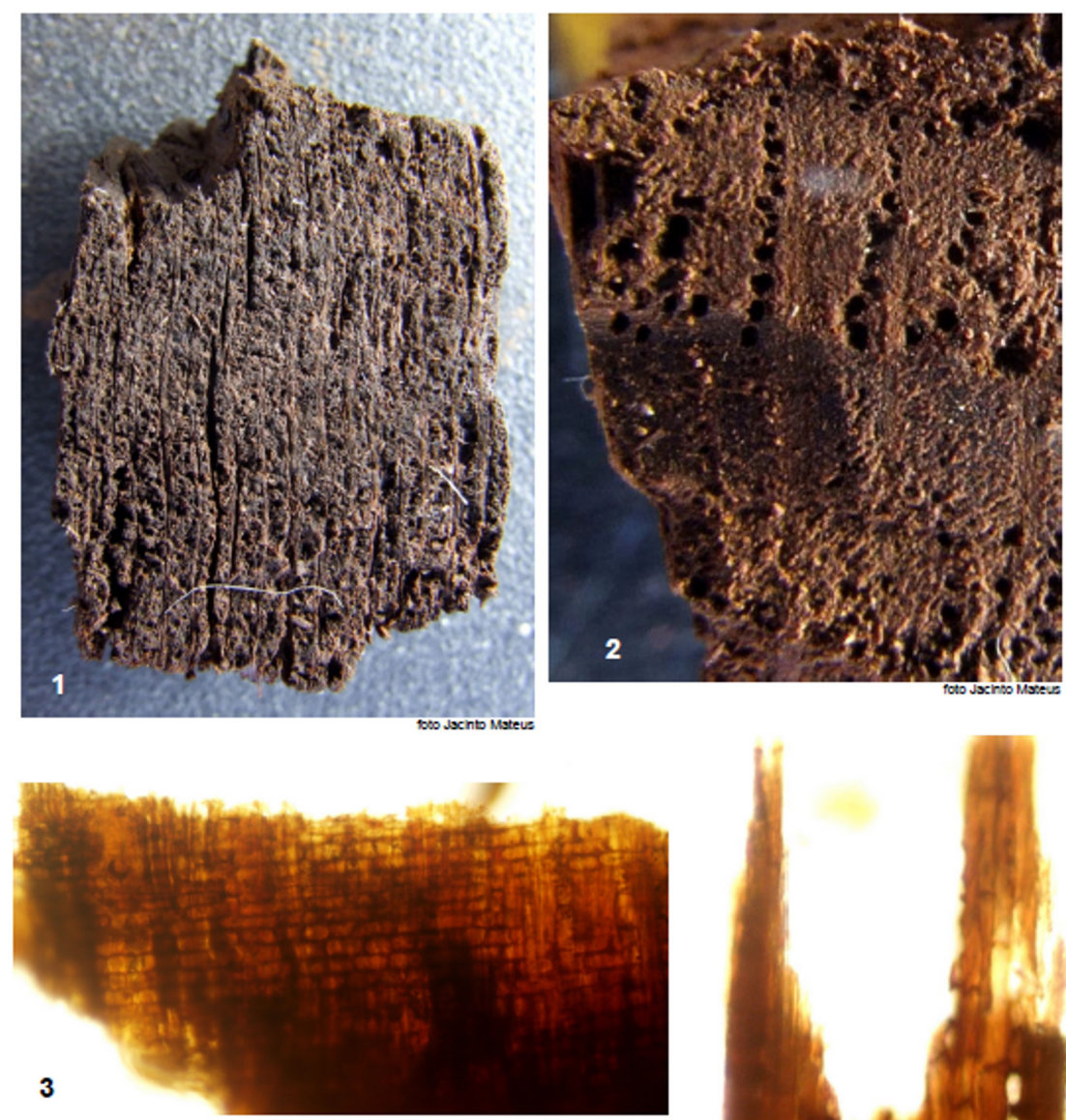

\section{Rio Tinto}

\section{Quercus suber}

1.Secção transversal;

2. Seç̧ão transversal, porosidae;

3. Secção radial, raio homogéneo;

4. Secção radial, pontuações intervasculares

5. Secção tangencial, raios unisseriados.

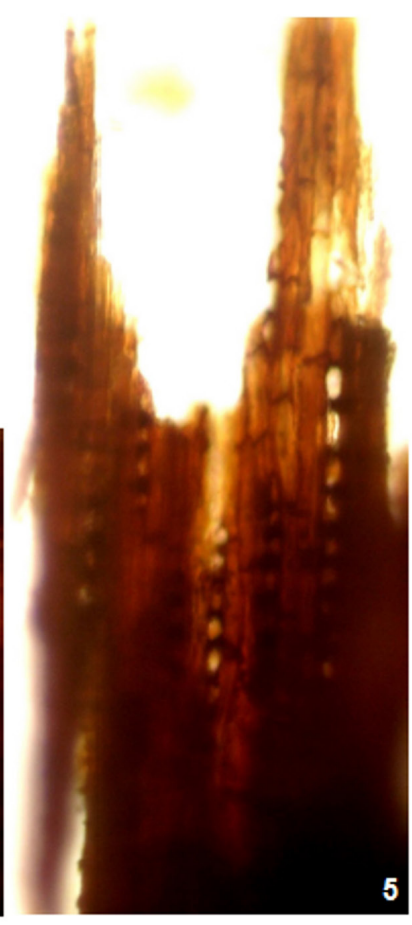

Figura 3 Muestras de madera de Riotinto. 
uma zona terminal, na madeira de Verão, praticamente sem poros indica tratar-se também de madeira de sobreiro.

O sobreiro é, como se sabe, uma árvore abundante em todo o sudoeste da Península Ibérica, com uma madeira muito dura e resistente, frequentemente utilizada em elementos construtivos. Em trabalho anterior (Rodríguez Trobajo, 2006) esta madeira já tinha sido identificada como uma das utilizadas neste tipo de rodas, designadamente em rodas encontradas nas minas de Rio Tinto e de S. Domingos. Além da madeira de sobreiro, também as madeiras de carvalho, de pinheiro bravo (Pinus pinaster) e silvestre (Pinus sylvestris), de abeto (Abies sp.), de lariço (Larix sp.), de freixo (Fraxinus angustifolia) e de faia (Fagus sylvati$c a$ ), têm sido identificadas como utilizadas na ma- nufatura de elementos constituintes destas rodas romanas de elevação de água. Deverá notar-se que as madeiras de abeto e de lariço foram identificadas em peças soltas e em raios da roda depositada no Museu de Huelva (Rodríguez Trobajo, 2006, 46).

\section{DATAÇÃo PELO RADIOCARBONO.}

As cinco amostras de madeira de sobreiro atrás referidas foram datadas fazendo uso do isótopo ${ }^{14} \mathrm{C}$ no Laboratório de Radiocarbono do Instituto Superior Técnico/Instituto Tecnológico e Nuclear, em Sacavém. As mostras eram constituidas por sequências de menos de10 anéis (Fig. 4). Foram todas sujeitas a um processo de descontaminação pela utilização sequencial de tratamentos com ácido $(\mathrm{HCl})$, base $(\mathrm{NaOH})$, ácido $(\mathrm{HCl})$, intercalados com lavagens com água desionizada até $\mathrm{pH}$ neutro (Soa-

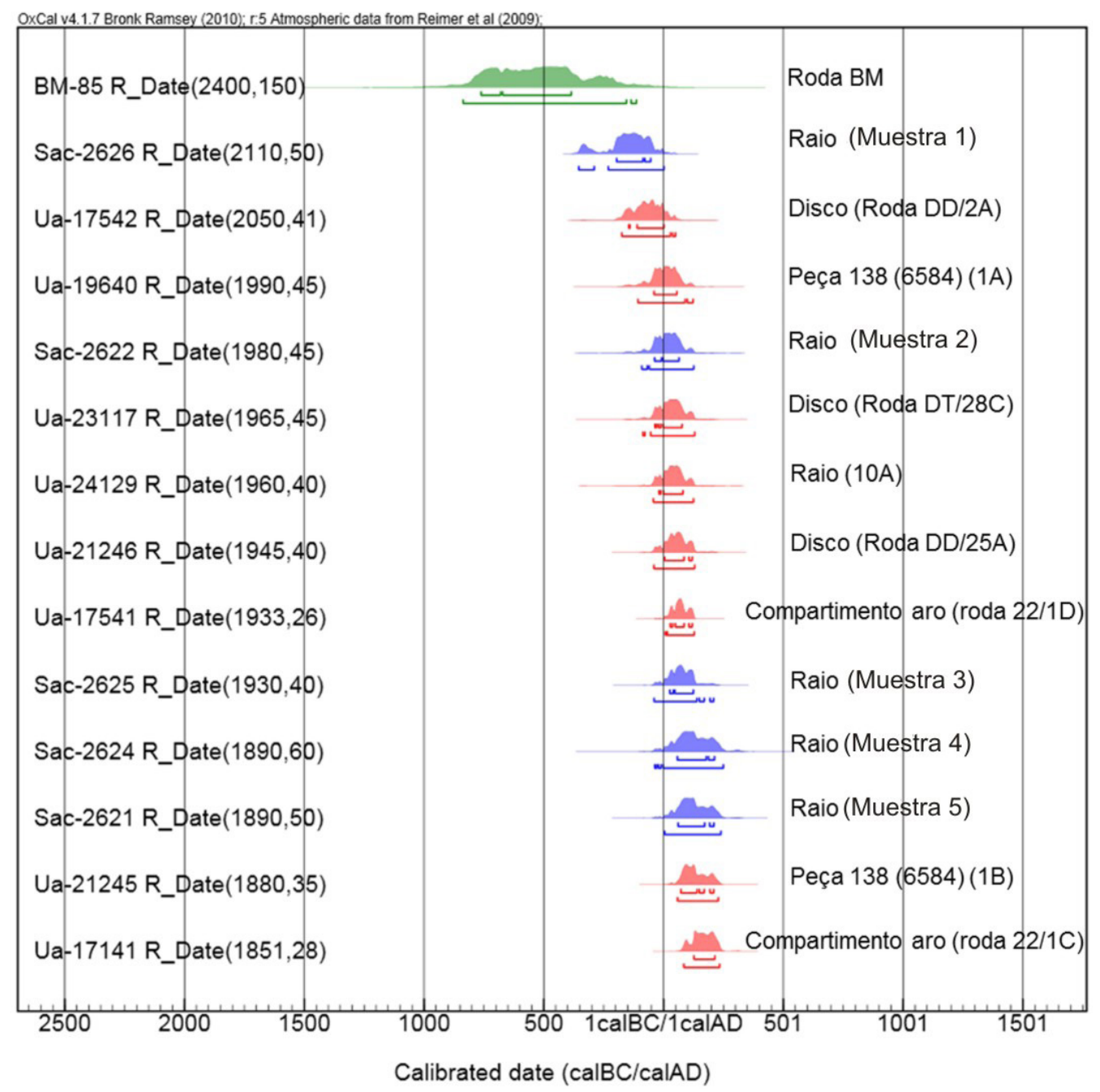

Figura 4 Dataciones de maderas de Norias de Riotinto. 


\begin{tabular}{|c|c|c|c|c|c|}
\hline $\begin{array}{l}\text { Ref. de } \\
\text { Lab. }\end{array}$ & $\begin{array}{c}\text { Ref. da } \\
\text { Amostra }\end{array}$ & $\begin{array}{l}\delta^{13} \mathrm{C} \\
(\%)\end{array}$ & $\begin{array}{l}\text { Data } \\
\text { (BP) }\end{array}$ & Data & cal $B C / A D)$ \\
\hline $\begin{array}{l}\text { Sac- } \\
2626\end{array}$ & Muestra 1 & $-25,5$ & $2110 \pm 50$ & $\begin{array}{l}\text { 197-86 cal BC }(0,838952) \\
79-54 \text { cal BC }(0,161048)\end{array}$ & $\begin{array}{l}\text { 354-291 cal BC }(0,112179) \\
231 \text { cal BC-1 cal AD }(0,887821)\end{array}$ \\
\hline $\begin{array}{l}\text { Sac- } \\
2622\end{array}$ & Muestra 2 & $-26,0$ & $1980 \pm 45$ & $\begin{array}{c}38-27 \text { cal BC }(0,102188) ; \\
25-9 \text { cal BC }(0,142188) ; \\
3 \text { cal BC-65 cal AD }(0,755624)\end{array}$ & $\begin{array}{c}91-69 \text { cal BC }(=, 026748) ; \\
60 \text { cal BC- } 126 \text { cal AD }(0,973252)\end{array}$ \\
\hline $\begin{array}{l}\text { Sac- } \\
2625\end{array}$ & Muestra 3 & $-25,9$ & $1930 \pm 40$ & $\begin{array}{l}27-41 \text { cal AD }(0,153332) ; \\
48-93 \text { cal AD }(0,561462) ; \\
97-125 \text { cal AD }(0,282506)\end{array}$ & $\begin{array}{c}39 \text { cal BC-139 cal AD }(0,972363) ; \\
155-169 \text { cal AD }(0,013796) ; \\
195-209 \text { cal AD }(0,01384)\end{array}$ \\
\hline $\begin{array}{l}\text { Sac- } \\
2624\end{array}$ & Muestra 4 & $-26,2$ & $1890 \pm 60$ & $\begin{array}{l}59-178 \text { cal AD }(0,859472) ; \\
188-213 \text { cal AD }(0,140528)\end{array}$ & $\begin{array}{c}37-28 \text { cal BC }(0,008137) ; \\
24-10 \text { cal BC }(0,012276) ; \\
3 \text { cal BC- } 252 \text { cal AD }(0,979587)\end{array}$ \\
\hline $\begin{array}{l}\text { Sac- } \\
2621\end{array}$ & Muestra 5 & $-26,2$ & $1890 \pm 50$ & $\begin{array}{l}61-143 \text { cal AD }(0,744267) ; \\
147-172 \text { cal AD }(0,149582) ; \\
193-210 \text { cal AD }(0,106152)\end{array}$ & $\begin{array}{l}\text { 7-11 cal AD }(0,005409) ; \\
17-239 \text { cal AD }(0,994591)\end{array}$ \\
\hline
\end{tabular}

QUADRO I. Datação pelo radiocarbono raios de rodas romanas de elevação da água em madeira das minas de Rio Tinto.

res, 2005). As datas convencionais de radiocarbono obtidas, fazendo uso da técnica da espectrometria de cintilação líquida (Soares, 2005) e calculadas segundo as recomendações de Stuiver e Polach (1977), encontram-se no Quadro I, acompanhadas de alguns dados julgados pertinentes, designadamente da sua identificação e do valor do fraccionamento isotópico em ${ }^{13} \mathrm{C}$. Além disso, as datas convencionais de radiocarbono foram calibradas fazendo uso da curva de calibração IntCal09 (Reimer et al., 2009) e do programa CÁLIB 6.0.1 (Stuiver e Reimer, 1993), encontrando-se as datas calibradas com os respectivos intervalos de confiança (com indicação da respectiva probabilidade) também no Quadro I.

\section{DisCUSSÃO DOS RESULTADOS.}

Já anteriormente ocorreram tentativas de datação pelo radiocarbono das rodas de madeira romanas encontradas nas minas de Rio Tinto. A primeira tentativa ocorreu ainda nos primórdios da divulgação do método de datação pelo radiocarbono e teve lugar no laboratório do British Museum. Foi colhida uma amostra de madeira da roda que se encontra depositada naquele Museu e o resultado obtido, $2400 \pm 150 \mathrm{BP}$, com uma incerteza muito grande (= 150 anos), permitiu atribuir a sua manufactura à época romana ("allowing for probable age of timber before fabrication, radiocarbon result is quite consistent with a Romano-Spanish origin”), uma vez que "doubts had been cast as to its antiquity" (Barker and MacKey, 1961).

Mais recentemente, uma investigação sólida foi levada a cabo por Rodríguez Trobajo (2006), o qual não se limitou simplesmente a datar algumas amostras de diversos elementos constituintes das rodas, dado que as sujeitou a um estudo dendrocronológico prévio, o que originou uma fiabilidade acrescida para os resultados obtidos. Além disso, as datações foram determinadas fazendo uso da técnica de AMS, no laboratório de Uppsala, o que lhe permitiu não só utilizar amostras constituidas por um único anel e mais do que uma amostra do mesmo anel (maior precisão nos resultados), mas também datar dois anéis da mesma peça de madeira, separados por um número conhecido de anos (de anéis). Faz-se notar que Rodríguez Trobajo, a par- 
tir de dois discos de roda (rodas DD e DT), obteve sequências de 280 e 180 anéis (anos). No entanto, como em qualquer das peças amostradas/datadas se ignora quantos anéis (anos) existiriam entre o anel mais externo identificado na peça amostrada e o último anel de crescimento da árvore que originou a peça em causa, as datas obtidas, tal como a determinada pelo British Museum, não passam de terminii post quem para o fabrico e utilização das rodas das minas de Rio Tinto.

Também as datas por nós obtidas não se podem considerar, pelos motivos atrás expostos, senão como terminii post quem.

No Quadro II e na Fig. 4 encontram-se todas as datas conhecidas, obtidas até agora, sobre amostras de madeira de constituintes das rodas elevadoras de água das minas romanas de Rio Tinto. Para a calibração das datas convencionais de radiocarbono utilizou-se a mesma metodologia referida atrás, embora no Quadro II, apenas se indique o intervalo de calibração de maior probabilidade (para um intervalo de confiança de $2 \sigma$ ) e se corrija esse intervalo, para o caso das datas obtidas no laboratório de Uppsala, somando o número de anos (anéis) existentes entre o anel datado e o anel exterior da peça de madeira amostrada determinado por Rodríguez Trobajo (op. cit., p. 50). Por outro lado, a Fig. 4 foi construida fazendo uso do programa OxCal v4.1.7 (Bronk Ramsey, 2009), mas utilizando a mesma curva de calibração (IntCal09).

\begin{tabular}{|c|c|c|c|c|c|}
\hline Ref. de Lab. & Prov. da Amostra & $\begin{array}{l}\text { Data } \\
\text { (BP) }\end{array}$ & Data Calibrada $(2 \sigma)$ & $\begin{array}{l}\text { Anos } \\
\text { a } \\
\text { Somar }\end{array}$ & $\begin{array}{c}\text { Idade } \\
\text { (Terminus post quem) }\end{array}$ \\
\hline BM-85 & Roda BM & $2400 \pm 150$ & 833-154 cal BC & - & 833-154 cal BC \\
\hline Sac-2626 & Raio Muestra 1 & $2110 \pm 50$ & $231 \mathrm{cal} B C-1 \mathrm{cal} A D$ & - & 231 cal BC-1 cal AD \\
\hline Sac-2622 & Raio Muestra 2 & $1980 \pm 45$ & $60 \mathrm{cal} \mathrm{BC}-126 \mathrm{cal} A D$ & - & $60 \mathrm{cal} \mathrm{BC}-126 \mathrm{cal} \mathrm{AD}$ \\
\hline Sac-2625 & Raio Muestra 3 & $1930 \pm 40$ & $39 \mathrm{cal} \mathrm{BC}-139 \mathrm{cal}$ AD & - & 39 cal BC-139 cal AD \\
\hline Sac-2624 & Raio Muestra 4 & $1890 \pm 60$ & $3 \mathrm{cal} B C-252 \mathrm{cal}$ AD & - & $3 \mathrm{cal} \mathrm{BC}-252 \mathrm{cal} \mathrm{AD}$ \\
\hline Sac-2621 & Raio Muestra 5 & $1890 \pm 50$ & $17-239 \mathrm{cal} A D$ & - & $17-239 \mathrm{cal} \mathrm{AD}$ \\
\hline Ua-24129 & Raio (10A) & $1960 \pm 40$ & $43 \mathrm{cal} \mathrm{BC}-94 \mathrm{cal} A D$ & 38 & $5 \mathrm{cal} \mathrm{BC}-132 \mathrm{cal} \mathrm{AD}$ \\
\hline Ua-23117 & Disco (Roda DT/28C) & $1965 \pm 45$ & $54 \mathrm{cal} B C-130 \mathrm{cal} A D$ & 85 & $31-215 \mathrm{cal} \mathrm{AD}$ \\
\hline Ua-17542 & Disco (Roda DD/2A) & $2050 \pm 41$ & $174 \mathrm{cal} B C-28 \mathrm{cal} A D$ & 149 & $25 \mathrm{cal} \mathrm{BC}-177 \mathrm{cal} \mathrm{AD}$ \\
\hline Ua-21246 & Disco (Roda DD/25A) & $1945 \pm 40$ & $40 \mathrm{cal} B C-130 \mathrm{cal} A D$ & 58 & $18-188 \mathrm{cal} \mathrm{AD}$ \\
\hline Ua-19640 & Peça 138 (6584) (1A) & $1990 \pm 45$ & $106 \mathrm{cal} \mathrm{BC}-90 \mathrm{cal} A D$ & 108 & 2-198 cal AD \\
\hline Ua-21245 & Peça 138 (6584) (1B) & $1880 \pm 35$ & $60-229 \mathrm{cal} A D$ & 38 & $98-267 \mathrm{cal} \mathrm{AD}$ \\
\hline Ua-17141 & $\begin{array}{l}\text { Compartimento aro } \\
\text { (roda } 22 / 1 \mathrm{C} \text { ) }\end{array}$ & $1851 \pm 28$ & $116-234 \mathrm{cal} A D$ & 23 & $139-257 \mathrm{cal} \mathrm{AD}$ \\
\hline Ua-17541 & $\begin{array}{l}\text { Compartimento aro } \\
\text { (roda 22/1D) }\end{array}$ & $1933 \pm 26$ & $17-128 \mathrm{cal} A D$ & 79 & $96-207 \mathrm{cal} \mathrm{AD}$ \\
\hline
\end{tabular}

QUADRO II. Datas de radiocarbono para elementos de rodas romanas de madeira para elevação da água nas minas de Rio Tinto. 


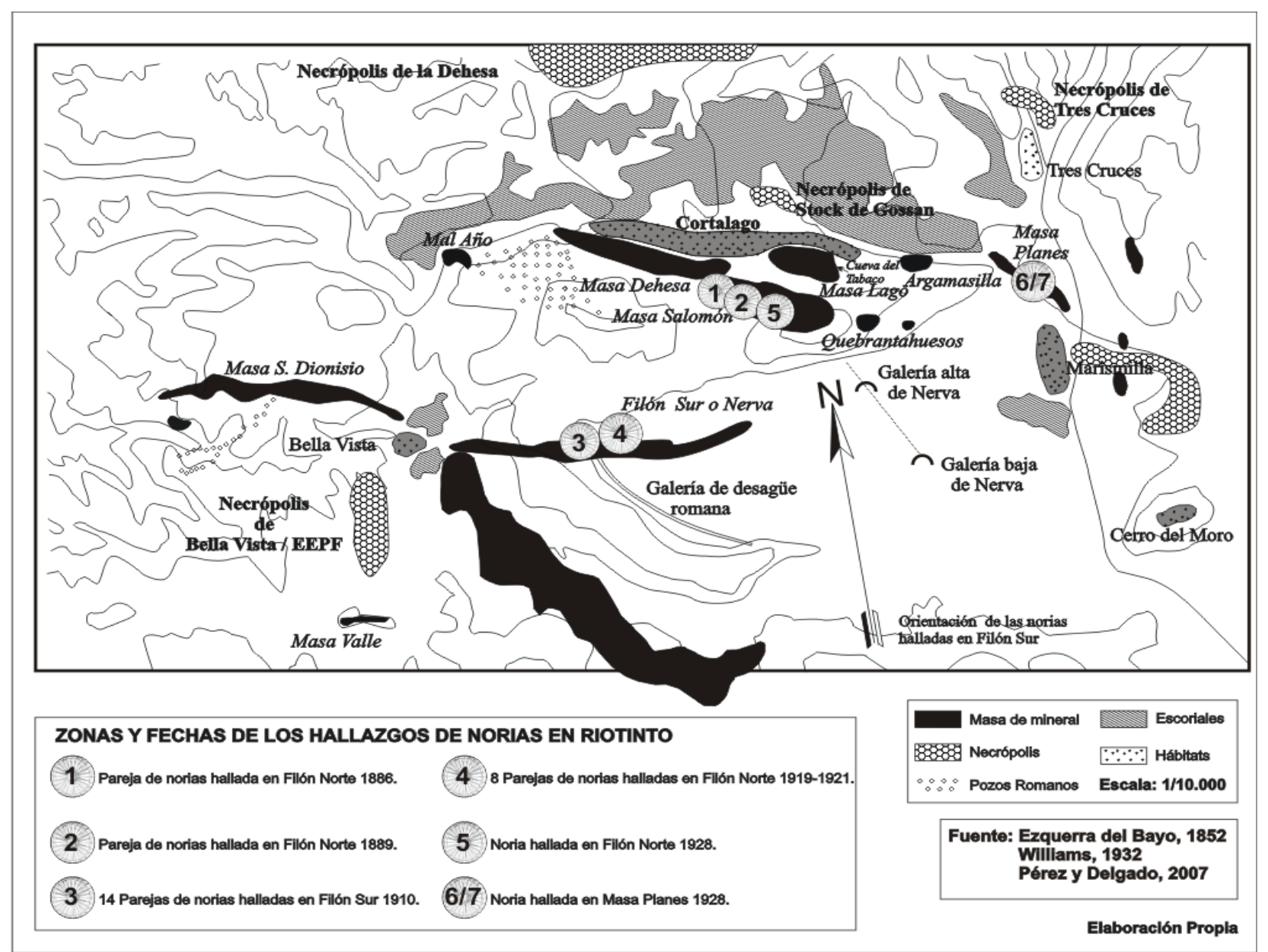

Plano 1: Labores mineras romanas en Riotinto, indicando el lugar y fecha de donde fueron halladas las ruedas hidráulicas. Los números 3 y 4 corresponden a las norias referidas en el presente trabajo.

Pela observação dos anéis de crescimento das amostras por nós datadas e sabendo que os raios, tem dimensões de comprimento um pouco menores que 2 metros, então estes teriam de ser obtidos a partir de secções longitudinais das árvores utilizadas. Além disso, segundo Rodríguez Trobajo (2006, p. 49), a madeira utilizada na sua manufatura, seria obtida num sector do cerne da árvore afastado do seu centro, a fim de evitar tensões que poderiam provocar a sua rotura. Embora se saiba que os sobreiros podem viver algumas centenas de anos, a análise das datas agora obtidas indiciam dois momentos ou, pelo menos, a utilização de madeiras com duas idades algo distintas - uma representada pela amostra Muestra 1 e outra pelas restantes amostras (a data obtida a partir da amostra Muestra 2 é estatisticamente distinta das restantes). Também a data obtida pelo British Museum, apesar do seu elevado desvio padrão, afasta-se das restantes.
A homogeneidade do desenho de construção das várias rotae encontradas nas minas romanas da faixa piritosa ibérica indiciam um mesmo momento para a sua construção ou momentos pouco afastados no tempo. De qualquer modo e uma vez que, como foi referido, as datas determinadas constituem terminii post quem para a construção das várias rodas que foram amostradas, não é possível afirmar, com os dados disponíveis se assim aconteceu ou se a sua construção se realizou em momentos diferenciados ao longo dos sécs. I a III d.C.

\section{REFERÊNCIAS}

Barker, H. y Mackey, J. (1961), "British Museum Natural Radiocarbon Measurements III", $R a$ diocarbon, 3, 39-45.

Bronk Ramsey, C. (2009), "Bayesian analysis of radiocarbon dates", Radiocarbon, 51/1, 337-360.

Davies, O. (1935), Roman Mines in Europe, Oxford. Delgado, A. y Mª C. Regalado (2010a), "Musealiza- 
ción del Patrimonio Minero en Riotinto (Huelva)", Patrimonio Geológico y Minero. Una apuesta por el desarrollo local sostenible, Huelva, 659-676.

(2010b), "Musealización del Patrimonio Minero de Riotinto (Huelva), Patrimonio Geológico y Minero. Una apuesta por el desarrollo local sostenible, Huelva, 677-693.

Domergue, C. (1987), Catalogue des mines et fonderies anticues de la Peninsule ibérique, Madrid.

Domergue, C.; Binet, C., y Bordes, J.H. (1999): "La roue de Sâo Domingos”, La revue. Musée des Arts et Métiers, 27, 49-59.

Domergue, C. y Bordes, J.H. 2004: "La roue élévatoire de la mine romaine de Tharsis (Huelva, Espagne). Étude archéologique et technique de ce type de roue", Problema de macchinismo in ambito romano. Machina idraulique nella letteratura técnica, nelle fonti stroriographiche e nelle evidente archeologiche di etá imperiale, Como, 87-105.

Ezquerra Del Bayo, J. (1852), Memoria sobre las minas de Río Tinto, Madrid.

Jones, G. D. B., Blakey, I. J.Y y Macpherson, E. C. F. (1960): "Dolaucothi: the Roman aqueduct", Bulletin of the Board of Celtic Studies, 19, 71-84 and plates III-V.

Luzón Nogué, J. M. (1968): “Los sistemas de desagüe en minas romanas del suroeste peninsular”, Archivo Español de Arqueología, XLI, 101-120.

Lewis, P. R. (1977), The Ogofau Roman gold mines at Dolaucothi, The National Trust Year Book.

Ojeda Calvo, R. (2006): "La Rota del Museo de Huelva: apuntes sobre el origen, adscripción, uso y funcionalidad de una rueda de evacuación de agua hallada en Minas de Riotinto", Rueda elevadora de agua de las minas de Riotinto: Memoria de Intervención, Cuadernos PH, 18, Consejería de Cultura de la Junta de Andalucía, Sevilla, 10-39.

Palmer, R. E. (1927): "Notes on some ancient mining equipments and systems", Transactions Institution of Mining and Metallurgy, XXXV, 299-336.

Pérez, J.A., Delgado, A. y Regalado, M.C. (2012): "Asentamiento romano de Marismilla (Riotinto-Nerva, Huelva)", Paisajes, Tiempos y $\mathrm{Me}$ moria (Pérez, J.A., Carriazo, J.L., y Gavilán, B., Eds), Huelva, 45-82.
Reimer, P.J., Baillie, M.G.L., Bard, E., Bayliss, A., Beck, J.W., Blackwell, P.G., Bronk Ramsey, C., Buck, C.E., Burr, G.S., Edwards, R.L., Friedrich, M., Grootes, P.M., Guilderson, T.P., Hajdas, I., Heaton, T.J., Hogg, A.G.; Hughen, K.A., Kaiser, K. F., Kromer, B., Mccormac, G., Manning, S., Reimer, R.W., Richards, D.A., Southon, J.R., Talamo, S.; Turney, C.S.M., Van Der Plicht, J., y Weyhenmeyer, C. E. (2009),"IntCal09 and Marine09 Radiocarbon Age Calibration Curves, 0-50,000 Years cal BP”, Radiocarbon, 51/4, 1111-1150.

Rodríguez Trobajo, E. (2006)"Material y cronología de las rotae del Museo de Huelva”, La rueda elevadora de agua de las minas romanas de Riotinto: memoria de intervención, Sevilla, 41- 61.

Schweingruber, F.H., (1990a), Anatomy of European Woods, Haupt.

(1990b), Mikroskopisch Holzanatomie. 3 auflege. Eidgenössische Forschungsanstalt für Wald, Schnee und Landschaft, Birmensdorf.

Slotta, R., Wollmann, V., y Dordea, I. 2002: Silber und Salz in Siebenbürgen, Bochum.

Stevenson, A.S. (1875): "Observations on a roman water Wheel from the ancient working of the mines of Tharsis in Southern Spain”, Archaeologia Eliana, VII, 276-281.

Soares, A.M.M. (2005), Variabilidade do "Upwelling" Costeiro durante o Holocénico nas Margens Atlânticas Ocidental e Meridional da Península Ibérica, Tese de Doutoramento, Faculdade de Ciências do Mar e do Ambiente, Universidade do Algarve, Faro.

Stuiver, M. y Polach, H.A. (1977), "Discussion. Reporting of ${ }^{14} \mathrm{C}$ Data", Radiocarbon, 19/3, 355363.

Stuiver, M. y Reimer, P.J. (1993), "Extended 14C database and revised CALIB radiocarbon calibration program", Radiocarbon, 35, 213-230.

Weisberger, G. (1979), "Das romische Wasserheberard aus Río Tinto in Spanien im British Museum London", Anschnitt, 2-3, 37-80.

Williams, D. (1932), Notes on Ancient History, and Museum exhibits at Rio Tinto, Archivo Fundación Riotinto, Inédito.

Wollmann, V. (1996), "Mineritul metalifer, extragerea sării şi carierele de piatră în Dacia romană”, Der Erzbergbau, die Salzgewinnung und die Steinbrüche im römischen Dakien, Cluj-Napoca. 
